\title{
Special Issue on Logics and Artificial Intelligence
}

\author{
Zoe Falomir · Vicent Costa · Enric Plaza · Karina \\ Gibert
}

Received: date / Accepted: date

\begin{abstract}
There is a significant range of ongoing challenges in Artificial Intelligence (AI) dealing with reasoning, planning, learning, perception and cognition, among others. In this scenario, many-valued logics emerge as one of the topics in many of the solutions to some of those AI problems.

This special issue presents a brief introduction to the relation between logics and AI and collects recent research works on logic-based approaches in AI.
\end{abstract}

Keywords logics, many-valued logics, logic programming, optimization, MaxSAT, MinSAT, calculus, completeness, planning, classifiers, explainable AI

\section{Introduction}

Artificial intelligence (AI) is the science and engineering devoted to the design of virtual and physical machines which intend to be able to perform intelligent tasks and/or activities

Zoe Falomir

University of Bremen

Faculty of Computer Science and Mathematics

Bremen Spatial Cognition Centre (BSCC)

E-mail: zfalomir@uni-bremen.de

Vicent Costa

Department of Philosophy, Universitat Autònoma de Barcelona (UAB)

Campus UAB, 08193, Bellaterra, Catalonia.

E-mail: vicente.costa@uab.cat

Enric Plaza

Artificial Intelligence Research Institute (IIIA-CSIC)

E-mail: enric@iiia.csic.es

Karina Gibert

Universitat Politècnica de Catalunya-BarcelonaTech

Subdirector of the Intelligent Data Science and Artificial Intelligence Centre

Vicedean of Big Data and Data Science and Artificial Intelligence at the Official Chamber of Informatics

Engineering in Catalonia

E-mail: karina.gibert@upc.edu 
involving other human faculties (e.g., association, perception or motor control) [5]. AI is today present in almost all aspects of daily life, drawing a lot of interest from governments, universities and societies in general. In AI research, classical logic has been widely used to solve challenges dealing with planning, learning, perception, and specially those focusing on knowledge representation and reasoning. Indeed, several approaches in AI literature show that the logic-based methodology has reached a high level of development. However, it is well established that classical Boolean logic is not enough when dealing with uncertainty, graded truth or similarity, among others, and therefore other more expressive logics appeared which became more relevant to AI. Non-classical logics emerged as an approach to solve many AI challenges. In fact, it was in the 20th century that the formalization of vagueness, ambiguity and uncertainty was addressed in logic. In this way, the idea of changing or removing some of the seven conventionally standard properties of classical logic led to the birth of non-classical logics. These logics include a wide class of different logic systems, such as modal logics, fuzzy logic, intuitionistic logic or many-valued logics $[24,27,7,8,25,31,20,10,12,13,11]$.

In the field of AI, let us recall that four general approaches are usually distinguished [33]: the logic-based AI, the non-logicist AI, the intelligent agent continuum approach, and the heterogeneous AI approach. The logic-based AI is a symbolic method that was concretely started by McCarthy [28] in 1959, and it is based on the general idea of using logic to formalize knowledge and solve problems by logical reasoning. Some of the main areas covered by this approach are knowledge representation, beliefs theory, system implementations, non-monotonic reasoning, abductive and inductive reasoning, commonsense reasoning, and planning and problem solving (for a general discussion on the logic-based AI the reader is referred to [29]). Notice that the logic-based AI is the approach tackled in this special issue.

The most prominent field of the applications of many-valued logic falls within logicbased AI. Some important areas of application are for example the automation of data and knowledge mining, the formalization of vague notions, and commonsense reasoning. In the literature, research works that use many-valued logics in AI include the following: Aksoy and Ercanoglu [1] used many-valued logics in landslide identification and classification; manyvalued logic for fuzzy control was reviewed and discussed by Moraga et al. [30]; Falomir et al. [22] used description logics to interpret digital images by describing each object by its colour and qualitative shape and by its main spatial features (location, relative orientation and topology) which allows to infer new object categories (e.g. doors) by reasoning; connections between weighted argumentation frames and t-norm-based logics have been explored by Corsi and Fermüller [14]; a fuzzy logic-based colour histogram analysis for discriminating benign skin lesions from malignant melanomas in dermoscopic images has been proposed by Almubarak et al. [2]; logic properties of relevant notions in fuzzy logic programming and computer science have been studied by Badia et al. [4] and Costa and Dellunde [16,15]; Eklund and Löfstrand [19] applied many-valued logics with the aim of enriching the language used in the manufacturing industry regarding information structure and its representation for products and production processes; Falomir and Pich [32] presented a logical approach to compose qualitative shapes and applied it to solve spatial reasoning tests; the relation between many-valued logic and decision theory under uncertainty has been analyzed by Flaminio et al. [23]; Falomir et al. [21] defined logics for combining a fuzzy-colour model with a Probabilistic Reference And GRounding mechanism (PRAGR) in order to obtain the most discriminative colour descriptor for an object depending on the context. And recently, a special issue on many-valued logics for reasoning has been presented by Dubois et al. [18]. 


\section{Context of this special issue}

This special issue was proposed in the 21 st edition of the International Conference of the Catalan Association for Artificial Intelligence (CCIA 2018), which took place on 8th-10th October 2018. The first edition of CCIA was in 1998, so it has been a long path, and in all this time, a rich community of researchers in AI have been well-established. This conference is organized by the Catalan Association for Artificial Intelligence (ACIA), which celebrates this year its 25th anniversary, and it is currently an international association which brings together researchers in the Catalan Countries and other countries around the world (i.e. Germany, Ireland, Sweden, etc.) gathering more than 200 members in 2019. ACIA is also a member society of the European Association for Artificial Intelligence (EurAI). The main goal of the CCIA conference is to maintain this well-established forum of researchers that allows international and interdisciplinary communication and collaboration among the Catalan and the International AI community. CCIA Proceedings have been published continuously since 2005 by IOS Press in the Artificial Intelligence Research and Development series. In recent editions of the CCIA conference, extended versions of the best papers were invited to be published in special issues of several journals. In this 21 st edition of CCIA, a special issue focused on the interactions of logics and applications of AI was organised, among others. Most of the papers presented in this issue are extended versions of papers that were presented at CCIA 2018 or research works developed by ACIA members.

\section{Papers in this Special Issue on Logics and Artificial Intelligence}

This special issue contains five papers, which are outlined in this section.

The paper titled Clause tableaux for maximum and minimum satisfiability [3] states that the inference systems proposed for solving SAT are unsound for solving MaxSAT and MinSAT because they preserve satisfiability but not the minimum and maximum number of clauses that can be falsified, respectively. And, in order to address this problem, the authors define: (1) a clause tableau calculus for MaxSAT and prove its soundness and completeness and (2) a clause tableau calculus for MinSAT and also prove its soundness and completeness. Finally, the authors also define a complete clause tableau calculus for solving both MaxSAT and MinSAT, in that the minimum number of generated empty clauses provides an optimal MaxSAT solution and the maximum number provides an optimal MinSAT solution.

The paper titled A Resolution Calculus for MinSAT [26] states that the logical calculus for SAT are not valid for MaxSAT and MinSAT because they preserve satisfiability but not the number of unsatisfied clauses. To address this issue, a MaxSAT resolution rule preserving the number of unsatisfied clauses was defined in the literature. This rule is complete for MaxSAT when it is applied following a certain strategy. In this paper, the authors first prove that the MaxSAT resolution rule also provides a complete calculus for MinSAT if it is applied following the strategy proposed here. Then an exact variable elimination algorithm for MinSAT is described based on that rule. Finally, the authors show how the results for Boolean MinSAT can be extended to solve the MinSAT problem of the multiple-valued clausal forms known as Signed CNF formulas.

The paper titled Relaxing Non-interference Requirements in Parallel Plans [6] explains that the aim of being able to reason about quantities, time or space has been the main objective of the many efforts on the integration of propositional planning with extensions to 
handle different theories. Planning Modulo Theories (PMT) is an approximation inspired by Satisfiability Modulo Theories (SMT) that generalizes the integration of arbitrary theories with propositional planning. Parallel plans are crucial to reduce plan lengths and hence the time needed to reach a feasible plan in many approaches. Parallelization of actions relies on the notion of (non-)interference, which is usually determined syntactically at compilation time. In this paper, the authors define a semantic notion of interference between actions in PMT. Apart from being strictly stronger than any syntactic notion of interference, the authors show how semantic interference can be easily and efficiently checked by calling an off-the-shelf SMT solver at compile time, constituting a technique orthogonal to the solving method.

The paper titled Solving Highly Cyclic Distributed Optimization Problems without Busting the Bank: a Decimation-based Approach [9] states that, in the context of solving large distributed constraint optimization problems (DCOP), belief-propagation and incomplete inference algorithms are candidates of choice. However, in general, when the problem structure is very cyclic, these solution methods suffer from bad performance, due to nonconvergence and many exchanged messages. So, in order to improve performances of the MaxSum inference algorithm when solving cyclic constraint optimization problems, this paper proposes to take inspiration from the belief-propagation guided decimation used to solve sparse random graphs (k-satisfiability). The authors propose the novel DeciMaxSum method, which is parameterized in terms of policies to decide when to trigger decimation, which variables to decimate, and which values to assign to decimated variables. Based on an empirical evaluation on a classical constraint optimization benchmarks (graph coloring, random graph, and Ising model), some of these combinations of policies, using periodic decimation, cycle detection-based decimation, parallel and non-parallel decimation, random or deterministic variable selection, and deterministic or random sampling for value selection, outperform state-of-the-art competitors in many settings.

The paper titled The logical style painting classifier based on Horn clauses and explanations ( $\ell$-SHE) [17] presents a logical Style painting classifier based on evaluated Horn clauses, qualitative colour descriptors and Explanations ( $\ell$-SHE). Three versions of $\ell$-SHE are defined, using rational Pavelka logic, and expansions of Gödel logic and product logic with rational constants: RPL, $G(\mathbb{Q})$ and $\sqcap(\mathbb{Q})$, respectively. The authors introduce a fuzzy representation of the more representative colour traits for the Baroque, the Impressionism, and the Post-Impressionism art styles. The $\ell$-SHE algorithm has been implemented in Swi-Prolog and tested on 90 paintings of the QArt-Dataset and on 247 paintings of the Paintings-91-PIB dataset. The percentages of accuracy obtained in the QArt-Dataset for each $\ell$-SHE version are: 73.3\% (RPL), 65.6\% $(G(\mathbb{Q}))$ and 68.9\% $(\sqcap(\mathbb{Q}))$. Regarding the Paintings-91-PIB dataset, the percentages of accuracy obtained for each $\ell$-SHE version are: $60.2 \%(\mathrm{RPL}), 48.2 \%(G(\mathbb{Q})$ ) and $57.0 \%(\sqcap(\mathbb{Q}))$. The authors' logic definition for the Baroque style has obtained the highest accuracy in both datasets, for all the $\ell$-SHE versions (the lowest Baroque case gets $85.6 \%$ of accuracy). An important feature of the classifier is that it provides reasons regarding why a painting belongs to a certain style. The classifier also provides reasons about why outliers of one art style may belong to another art style, giving a second classification option depending on its membership degrees to these styles. 


\section{Epilog}

The guest editors of this Special Issue on Logics and Artificial Intelligence would like to thank all the authors who submitted articles to this issue. We are also grateful to the reviewers for their effort in evaluating the papers considered, and for giving highly constructive feedback to the authors. Finally, we also thank the general editors for their support.

\section{Acknowledgments}

Z. Falomir thanks the Cognitive Qualitative Descriptions and Applications (CogQDA) project funded by the University of Bremen and the YERUN Research Mobility Award (Young European Research UNiversities, second edition, 2018/2019). V. Costa is supported by the grant FI-2017 (Generalitat de Catalunya and the European Social Fund), by a YERUN Research Mobility Award (Young European Research UNiversities, first edition, 2017), by a grant Beca Fundación Universia para la formación de Doctores con Discapacidad 2018-2019, and by a DAAD Short-Term Grant, 2019 (57442045). V. Costa and Z. Falomir thank the support of the Bremen Spatial Cognition Center (BSCC). E. Plaza thanks the support by DIVERSIS (CSIC Intramural 201750E064). K. Gibert thanks the support of the Intelligent Data Science and Artificial Intelligence Research Center at UPC and the grant CO-2018-23-T-00454 funded by UPC to organize the 21st CCIA in Roses (Catalonia, Spain) in October 2019.

\section{References}

1. Aksoy, B., Ercanoglu, M.: Landslide identification and classification by object-based image analysis and fuzzy logic: An example from the Azdavay region (Kastamonu, Turkey). Computers \& Geosciences 38(1), 87-98 (2012). https://doi.org/10.1016/j.cageo.2011.05.010

2. Almubarak, H.A., Stanley, R.J., Stoecker, W.V., Moss, R.H.: Fuzzy color clustering for melanoma diagnosis in dermoscopy images. Information 8(3), 89 (2017). https://doi.org/10.3390/info8030089

3. Argelich, J., Li, C.M., Manyà, F., Soler, J.R.: Clause tableaux for maximum and minimum satisfiability. Logic Journal of the IGPL (08 2019). https://doi.org/10.1093/jigpal/jzz025, https://doi .org/10. 1093/jigpal/jzz025, jzz025

4. Badia, G., Costa, V., Dellunde, P., Noguera, C.: Syntactic characterizations of classes of first-order structures in mathematical fuzzy logic. Soft Comput. 23(7), 2177-2186 (2019). https://doi.org/10.1007/s00500019-03850-6

5. Boden, M.: AI: Its Nature and Future. Oxford University Press (2016)

6. Bofill, M., Espasa, J., Villaret, M.: Relaxing Non-interference Requirements in Parallel Plans. Logic Journal of the IGPL (2019)

7. Bolc, L., Borowik, P.: Many-Valued Logics 1: Volume 1: Theoretical Foundations. Many-valued Logics, Springer (1992)

8. Bolc, L., Borowik, P.: Many-valued logics 2 - automated reasoning and practical applications. Springer (2003)

9. Cerquides, J., Monet, R., Picard, G., RodrÍguez-Aguilar, J.A.: Solving Highly Cyclic Distributed Optimization Problems without Busting the Bank: a Decimation-based Approach. Logic Journal of the IGPL (2019)

10. Cignoli, R., d'Ottaviano, I., Mundici, D.: Algebraic Foundations of Many-Valued Reasoning. Trends in Logic, Springer Netherlands (2013)

11. Cintula, P., Fermueller, C., Noguera, C.: Handbook of Mathematical Fuzzy Logic. No. v. 3 in Handbook of Mathematical Fuzzy Logic, College Publications (2015), https://books.google.de/books?id= PZh0jwEACAAJ 
12. Cintula, P., Hájek, P., Noguera, C.: Handbook of Mathematical Fuzzy Logic - volume 1. No. 37 in Studies in Logic, Mathematical Logic and Foundations, College Publications, London (2011)

13. Cintula, P., Hájek, P., Noguera, C.: Handbook of Mathematical Fuzzy Logic - volume 2. No. 38 in Studies in Logic, Mathematical Logic and Foundations, College Publications, London, petr cintula, petr hájek, carles noguera edn. (2011)

14. Corsi, E.A., Fermüller, C.G.: Connecting fuzzy logic and argumentation frames via logical attack principles. Soft Comput. 23(7), 2255-2270 (2019). https://doi.org/10.1007/s00500-018-3513-2

15. Costa, V., Dellunde, P.: On the existence of free models in fuzzy universal horn classes. J. Applied Logic 23, 3-15 (2017). https://doi.org/10.1016/j.jal.2016.11.002

16. Costa, V., Dellunde, P.: Term models of horn clauses over rational pavelka predicate logic. In: 47th IEEE International Symposium on Multiple-Valued Logic, ISMVL 2017, Novi Sad, Serbia, May 22-24, 2017. pp. 112-117. IEEE Computer Society (2017). https://doi.org/10.1109/ISMVL.2017.26

17. Costa, V., Dellunde, P., Falomir, Z.: The logical style painting classifier based on Horn clauses and explanations. Logic Journal of the IGPL (2019)

18. Dubois, D., Esteva, F., Flaminio, T., Noguera, C., Prade, H., Rodríguez, R.O.: Many-valued logics for reasoning: Essays in honor of Lluís Godo on the occasion of his 60th birthday. Soft Comput. 23(7), 2125-2127 (2019)

19. Eklund, P., Löfstrand, M.: Many-valued logic in manufacturing. In: Ganzha, M., Maciaszek, L.A., Paprzycki, M. (eds.) Position Papers of the 2016 Federated Conference on Computer Science and Information Systems, FedCSIS 2016, Gdańsk, Poland, September 11-14, 2016. Annals of Computer Science and Information Systems, vol. 9, pp. 11-17 (2016). https://doi.org/10.15439/2016F73

20. Esteva, F., Godo, L.: Monoidal t-norm based logic: towards a logic for left-continuous t-norms. Fuzzy Sets and Systems 124(3), 271-288 (2001). https://doi.org/10.1016/S0165-0114(01)00098-7

21. Falomir, Z., Costa, V., Gonzalez-Abril, L.: Obtaining discriminative colour names according to the context: Using a fuzzy colour model and probabilistic reference grounding. International Journal of Uncertainty, Fuzziness and Knowledge-Based Systems 27(1), 107-142 (2019). https://doi.org/10.1142/S0218488519400063

22. Falomir, Z., Jiménez-Ruiz, E., Escrig, M.T., Museros, L.: Describing images using qualitative models and description logics. Spatial Cognition \& Computation 11(1), 45-74 (2011)

23. Flaminio, T., Hosni, H., Lapenta, S.: Convex mv-algebras: Many-valued logics meet decision theory. Studia Logica 106(5), 913-945 (2018). https://doi.org/10.1007/s11225-016-9705-9

24. Gottwald, S.: Fuzzy sets and fuzzy logic: the foundations of application - from a mathematical point of view. Artificial intelligence, Vieweg (1993)

25. Hájek, P.: Metamathematics of Fuzzy Logic. Trends in Logic, Springer Netherlands (1998)

26. Li, C.M., Xiao, F., Manyà, F.: A Resolution Calculus for MinSAT. Logic Journal of the IGPL (2019)

27. Malinowski, G.: Many-Valued Logics. Oxford University Press (1993)

28. McCarthy, J.: Programs with common sense. In Proceedings of the Teddington Conference on the Mechanization of Thought Processes. London: Her Majesty’s Stationery Office. pp. 756-791 (1959)

29. Minker, J.: Logic-Based Artificial Intelligence. The Springer International Series in Engineering and Computer Science, Springer US (2012)

30. Moraga, C., Trillas, E., Guadarrama, S.: Multiple-valued logic and artificial intelligence fundamentals of fuzzy control revisited. Artif. Intell. Rev. 20(3-4), 169-197 (2003). https://doi.org/10.1023/B:AIRE.0000006610.94970.1d

31. Novák, V., Perfilieva, I., Močkoř, J.: Mathematical Principles of Fuzzy Logic. The Springer International Series in Engineering and Computer Science, Springer US (2012), https://books.google.de/ books?id=uzPjBWAAQBAJ

32. Pich, A., Falomir, Z.: Logical composition of qualitative shapes applied to solve spatial reasoning tests. Cognitive Systems Research 52, 82-102 (2018). https://doi.org/10.1016/j.cogsys.2018.06.002

33. Russell, S., Norvig, P.: Artificial Intelligence: A Modern Approach. Always learning, Pearson (2016), https://books.google.es/books?id=XS9CjwEACAAJ 